\title{
Fabrication System for Large-Area Seamless Nanopatterned Cylinder Mold Using the Spiral Laser Interference Exposure Method
}

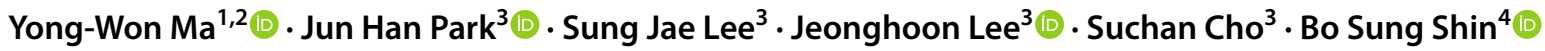

Received: 6 September 2021 / Revised: 23 December 2021 / Accepted: 13 January 2022 / Published online: 21 February 2022

(c) The Author(s) 2022

\begin{abstract}
With the advancement in the field of nanotechnology, nanopatterning finds extensive application not only in high valueadded products but also in inexpensive products. In addition, the technology required for the mass production of inexpensive products, such as the continuous roll-to-roll (R2R) process, is rapidly emerging. Extensive research has been conducted on the manufacture of submicron- and nano- molds. In this study, we have proposed a laser interference exposure for fabricating nanopatterned cylindrical molds that can be used in continuous roll-to-roll patterning. Additionally, we have demonstrated spiral exposure process to fabricate a seamless patterning on a cylinder (length of $300 \mathrm{~mm}$ and diameter of $100 \mathrm{~mm}$ ) using a prism. The pattern was transferred to the flat mold using UV resin and measured using a field emission scanning electron microscope; the pattern was measured to have a uniform with nano pattern line width $(75 \mathrm{~nm})$ and a sub-micron period $(286 \mathrm{~nm})$. It was observed that the proposed method for fabrication of the roll mold using laser interference lithography is a fast and reliable seamless patterning.
\end{abstract}

Keywords Cylindrical mold $\cdot$ Laser interference lithography $\cdot$ Nanofabrication $\cdot$ Seamless patterning $\cdot$ Nano-transfer

\section{Introduction}

In recent years, nanotechnology has developed rapidly with a focus on developing cost-effective methods for the fabrication of nanoscale patterns for large areas [1-20]. Nanoimprint lithography (NIL) is a low-cost and largearea processing method for fabricating nanopatterns. It can be classified into two types, thermal NIL and ultraviolet (UV) curable NIL and flat or cylindrical based on the type

Yong-Won Ma, Jun Han Park and Sung Jae Lee contributed equally to this work.

Bo Sung Shin

bosung@ pusan.ac.kr

1 Interdisciplinary Department for Advanced Innovative Manufacturing Engineering, Pusan National University, Busan, Korea

2 Department of Policy Planning, Ulsan Technopark, Ulsan, Korea

3 Department of Cogno-Mechatronics Engineering, Pusan National University, Busan, Korea

4 Department of Optics and Mechatronics Engineering, Pusan National University, Busan, Korea of mold. It is easier to produce a flat mold using flat-plate NIL as compared to roll-to-plate and roll-to-roll; however, continuous production is impossible using flat-plate NIL. Therefore, research on roller-type imprint processes, namely the roll-to-roll and roll-to-plate processes is actively ongoing $[1-18,20,21]$. However, the seamless fabrication of micro/nanostructures using a cylindrical mold is an area of concern. Conventionally, the manufacturing of cylindrical molds includes a wrapping process that involves attaching polymers or nickel plate molds fabricated using other conventional processes. Fabricating a nanopatterned mold on a flat plate and wrapping it around a cylinder is easier than directly patterning on the cylinder. However, each of these processes has several drawbacks. The wrapping process inevitably results in a seam; consequently, it is difficult to implement a continuous roll-to-roll imprinting process. Hence, various researchers have studied the direct patterning process on cylinders without wrapping using electron beam lithography [6-10], photolithography [12-14], and ablation using a pulsed laser $[15,16]$. Electron beam lithography can produce precise nanopatterns; however, this process is implemented in a high-vacuum environment, which is expensive and technically difficult to establish. Although the laser-ablation method is fast and simple, fabricating a 
pattern of accurate size is difficult due to the non-constant laser peak power. Moreover, producing a line width of less than $500 \mathrm{~nm}$ is difficult owing to heat transfer by the photothermal effect. Adequate research on conventional photolithography has been conducted [12-14]; however, the resolution that has been adopted is not sufficient for nanofabrication. Herein, we investigate an alternative process known as laser-interference lithography (LIL) to produce nanopatterns. This process relies on the interference phenomenon of light. Theoretically, the period of the 2-beam interference is equal to half the wavelength of the beam. However, it is practically impossible to expose two beams at $180^{\circ}$; hence, we aim to create a pattern whose period is slightly greater than half the wavelength. LIL can quickly fabricate both micro and nanopatterns over a relatively large area $[18,19$, 22-30]. In addition, the construction costs for LIL are lower than those for e-beam lithography systems. Thus, micropatterns and nanopatterns can be quickly produced using the laser interference pattern. Various methods, such as Lloyd's mirror [22, 23], beam splitter [18, 19, 24-26], phase mask [27], and prism-based laser interference [28-30] have been investigated. The Lloyd's mirror method performs interference exposure in a basic manner; however, both the interference angle and structure period are fixed in this method. In addition, the substrate can get damaged by the mirror in contact with the substrate. The beam splitter method is the most popular among the existing LIL exposure methods and can produce patterns of various sizes by adjusting the angle of the beams. However, this method requires a massive space, the optical system becomes complicated, and an accurate exposure point is required. The phase-mask method is used to split the laser beam into primarily two first-order beams for laser interference exposure. However, this method involves unnecessary exposure to zero, second, or higherorder beams, which affects the pattern quality. Prism-based interference exposure can create patterns of various periods using a basic Lloyd's mirror device. However, the prism must be changed for change of target pattern. Despite some obvious limitations and challenges, attempts have been made to use the aforementioned methods to fabricate nanopatterned cylinders.

When we design a cylinder-shaped large-area seamless nanopattern printing system, we mainly consider three factors: pattern distortion due to cylindrical curvature, interference exposure method based on prism, and pattern errors due to overlapping exposures. First, we concluded that distortion due to curvature could be neglected when the diameter is more than $100 \mathrm{~mm}$ [31]. Second, we confirmed that various patterns could be fabricated using the LIL system with the prism [17]. Finally, the error in pattern could occur if overlapped during exposure [14], and based on this result, it was important that the distance between the prism and cylinder be kept constant. However, because the above three studies were conducted only in a small area $(\sim 50 \mathrm{~mm})$, and we considered that a third factor can cause defects, we designed a fabrication system based on the above three results for large-area nanopatterned cylinders.

\section{Laser-Interference Lithography (LIL) System for Micro/Nano Cylindrical Molds}

\subsection{Laser-Optical System}

An optical system to fabricate nanoroll molds using laser interference exposure was developed as shown in Fig. 1a. Continuous wave $(\mathrm{CW})$ laser is used to fabricate the interference pattern on a photoresist (PR). In laser-interference exposure, gas lasers such as $\mathrm{HeNe}$ lasers have been used due to the coherence length. However, in recent years, inexpensive and compact semiconductor lasers have been increasingly used owing to their low maintenance costs and small space requirements. In this study, we have used a $360 \mathrm{~nm}$ UV solid-state laser (MSLFN-360, Changchun New Industries Optoelectronics Technology Co., Ltd., Changchun, China), and a heat sink (TC-01, Changchun New Industries Optoelectronics Technology Co., (a)

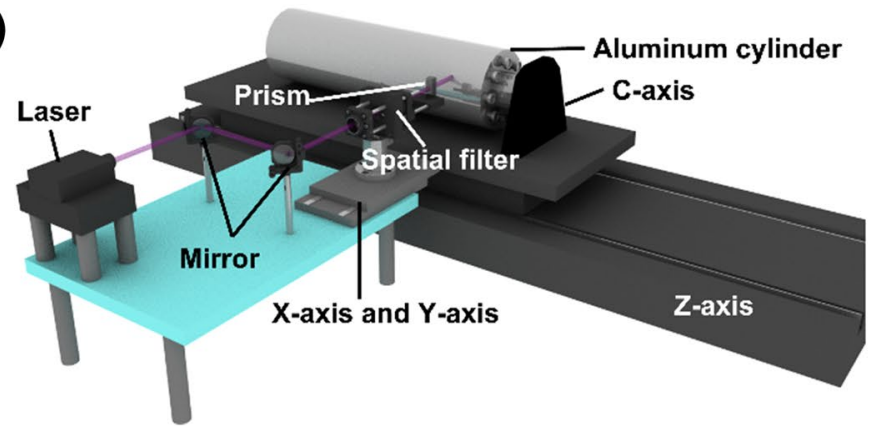

(b)

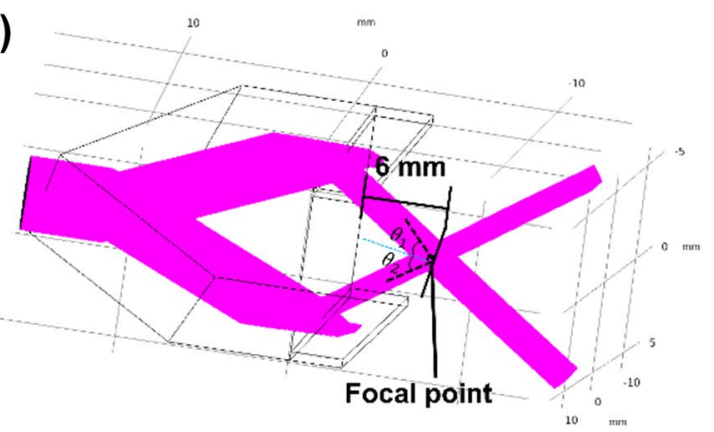

Fig. 1 Cylinder-shaped large-area laser nanopatterning system: a System overview, b 3D ray tracing of the prism 
Table 1 Laser specifications

\begin{tabular}{ll}
\hline Wavelength $(\mathrm{nm})$ & 360 \\
Operating mode & Continuous wave \\
Average out power $(\mathrm{mW})$ & 30 \\
Transverse mode & TEM $_{00}$ \\
Longitudinal mode & Single \\
Coherent length $(\mathrm{m})$ & $>50$ \\
Spectral linewidth $(\mathrm{nm})$ & $<0.00001$ \\
\hline
\end{tabular}

Ltd., Changchun, China) is used to obtain a stable laser output. The laser specifications are presented in Table 1.

To achieve a compact system, the laser beam was aligned in a perpendicular direction. Typically, laser-beam transmission involves the use of mirrors or optical fibers. With mirrors, the laser power loss is insignificant. However, to transmit the beam accurately, it is essential to control many mirrors. Mirrors are also affected by airborne particles, such as dust and fine particles that can damage the optical components. Hence, the optical system must be placed in an environment that prevents the inflow of particles. In contrast, using fiber enables the laser beam to be transmitted in free space; thus, a more flexible system can be configured and unlike mirror transmission, the influence of particles in air is insignificant. However, a singlemode fiber is required to obtain a clear interference pattern and it results in inevitable and significant power loss due to coupling efficiency. In our previous research, we had proposed a method to manufacture a pattern by connecting optical fibers to an interference-exposure box that included a prism [17]. However, the total processing time increased due to reduced power. Herein, we investigated a mirror beam-transmission system to implement an efficient and fast process by reducing the total exposure time. The mirror-aligned beam is passed through a spatial-filter system (Fig. 1a) to produce a coherent beam for the laser-interference exposure. A prism is used to divide the beam into two parts, and an interference phenomenon is generated at the intersection of the beams. The period of the two-beam interference pattern is expressed as follows:

$\Lambda=\frac{\lambda}{n \cdot\left(\sin \theta_{1}+\sin \theta_{2}\right)}$,

where $\Lambda$ is the period of the two-beam interference pattern, $\lambda$ is the wavelength of the light source, $n$ is the refractive index of the medium, and $\theta_{1}$ and $\theta_{2}$ are the angles of the two beams with the surface. The two beams split through the prism are at the same angle. The beam shape was gaussian, but it become rectangular with a width of $2 \mathrm{~mm}$ and height of $4 \mathrm{~mm}$ after passing through the prism (Fig. S1). Figures $1 \mathrm{~b}$ show the optical path of the beam passing through the prism. When the beam is passing through the prism, $\theta_{1}$ and $\theta_{2}$ is approximately $39^{\circ}$, and the distance between the prism and substrate is $6 \mathrm{~mm}$. If the distance between the prism and the cylinder surface is lower or higher than the focal point (distance between the prism and substrate is $6 \mathrm{~mm}$ ), the split beams do not meet each other completely, so there is a part where interference does not occur, and as a result, a seam is created.

The proposed system includes four axis stages that can move the cylinder and optics, making it possible to fabricate reliable patterns. The cylinder is used in motion by a linear Z-axis stage (LS-270, Physik Instrumente, Karlsruhe, Germany) and rotation stage (L-611.94AD, Physik Instrumente, Karlsruhe, Germany) whereas the optical components which include the laser-displacement sensor, prism and spatial filter system are used in motion by the $\mathrm{X}$-axis stage (N-565.260, Physik Instrumente, Karlsruhe, Germany) and Y-axis stage (M-VP-5ZA, Newport, California, USA). A laser displacement sensor (CD22-15-485, Optex FA Co., Ltd., Kyoto, Japan) is used to measure the distance between the cylinder and prism. The wavelength of the laser-displacement sensor is $650 \mathrm{~nm}$, and it was experimentally confirmed that the light source of the sensor did not react to a PR. Similar to a computer numerical control lathe, the $\mathrm{X}$-axis and $\mathrm{Y}$-axis stages are moved to align the center and compensate distance between the prism and cylinder using the measured distance during cylinder rotating (Fig. 2).

\subsection{Experimental Conditions}

The cylinder is made of an aluminum alloy 7075 (length $=350 \mathrm{~mm}$; diameter $=100 \mathrm{~mm}$ ) and plated with $\mathrm{H}-\mathrm{Cr}$ (thickness, $100 \mu \mathrm{m}$ ). In general, positive PRs yield better resolution than negative PRs; therefore, positive PRs are more suitable for producing submicron or nanopatterns. In this study, we have used the ma-P 1205 PR (Microresist Technology GmbH, Berlin, Germany). The aluminum roll was coated with PR using the droplet method, and instead of the soft bake process, the cylinder was rotated for 2 days at room temperature to evaporate the solvent in the PR. A spiral exposure process was performed to facilitate seamless

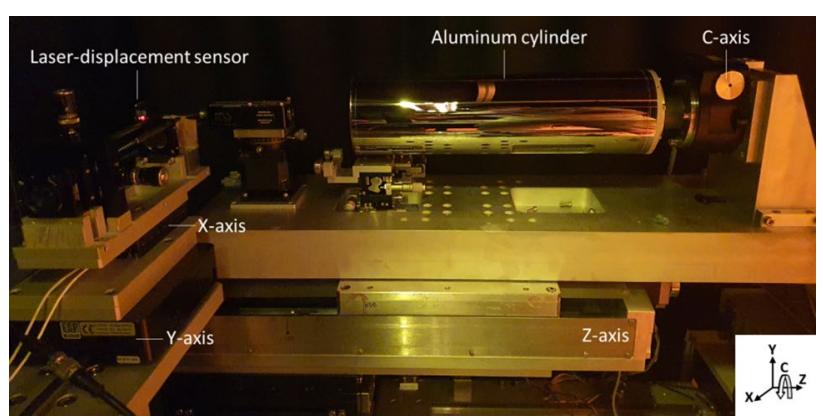

Fig. 2 Image of the various stages for a laser-interference exposure system 
fabrication (Fig. 3a). The rotation value is set to $9 \%$ s and the $\mathrm{Z}$-axis stage is set to move at $0.05 \mathrm{~mm} / \mathrm{s}$. The total exposure time is $1.7 \mathrm{~h}$. A pattern is generated by dipping the exposed cylinder in ma-D 377 (Microresist Technology GmbH, Berlin, Germany) for $10 \mathrm{~s}$. The roll mold is then baked at $100{ }^{\circ} \mathrm{C}$ for $1 \mathrm{~h}$ to stabilize the patterns. To observe the pattern formed on the cylindrical mold, the one-dimensional interference pattern formed on the cylinder was duplicated using a UV-curable resin (MINS 511RM, Minuta Technology Co. Ltd., Cheongju, Korea) on polyester (PET) film. The pattern replicated on the PET film was measured using the field emission scanning electron microscope (FE-SEM).

\section{Results and Discussion}

We fabricated a continuous pattern with a length of $300 \mathrm{~mm}$ on the cylinder. Figure 4a shows a photograph of the seamless roll mold that highlights the pattern on the cylindrical surface. The conventional patch-to-patch method consists of a rotation step and a Z-axis moving step. In this process, after a $360^{\circ}$ rotation, the laser $\mathrm{Z}$-axis is moved along the beam width, and the steps are repeated. However, the conventional patch-to-patch method is not suitable for seamless nanofabrication because the positioning resolution of the
Z-axis stage can cause unintended gaps or overlap between beams. In microfabrication, position precision is not a critical issue; however, in nanofabrication, it can cause defects as shown in Fig. 3b. Therefore, we applied a continuous spiral exposure process instead of a step-by-step process. To produce a seamless pattern, the c-axis and z-axis stages are moved simultaneously, and when the c-axis rotates once, it must move as much as the beam width size along the z-axis. Since the rotation speed is designed at $9 \% \mathrm{~s}$, and the z-axis is moved at $0.05 \mathrm{~mm} / \mathrm{s}$, the cylinder moves $2 \mathrm{~mm}$ while rotating once for $40 \mathrm{~s}$. The rotation speed per second is closely related to the exposure intensity, and the total exposure amount was not allowed to exceed $30 \mathrm{~mJ} / \mathrm{cm}^{2}$.

At all six points (Fig. 4b-g), the period is approximately $286 \mathrm{~nm}$, and the line width is approximately $75 \mathrm{~nm}$ (concave part in the image). It was confirmed that the pattern period obtained as a result of the experiment is the same as that theoretically calculated by Eq. (1). The images reveal that the patterns of periods are mostly uniform because the interference angle is uniformly irradiated on the surface owing to the use of the prism. However, it is evident that the difference in depth between the patterns is not constant in the six images. This is because of three reasons: the first is the nonuniformity of the exposure intensity. Given the Gaussian intensity distribution of the laser, a pattern with a certain (a) Continuous spiral exposure
(simultaneous rotation and Z-axis move)

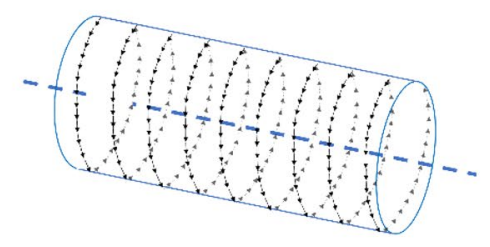

(b)

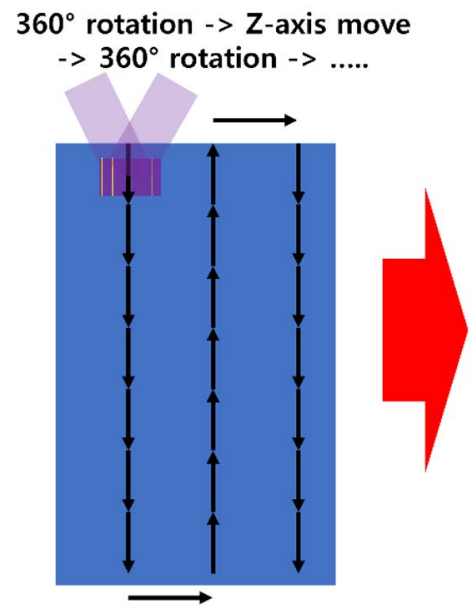

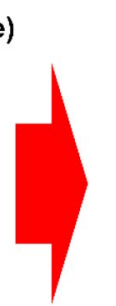
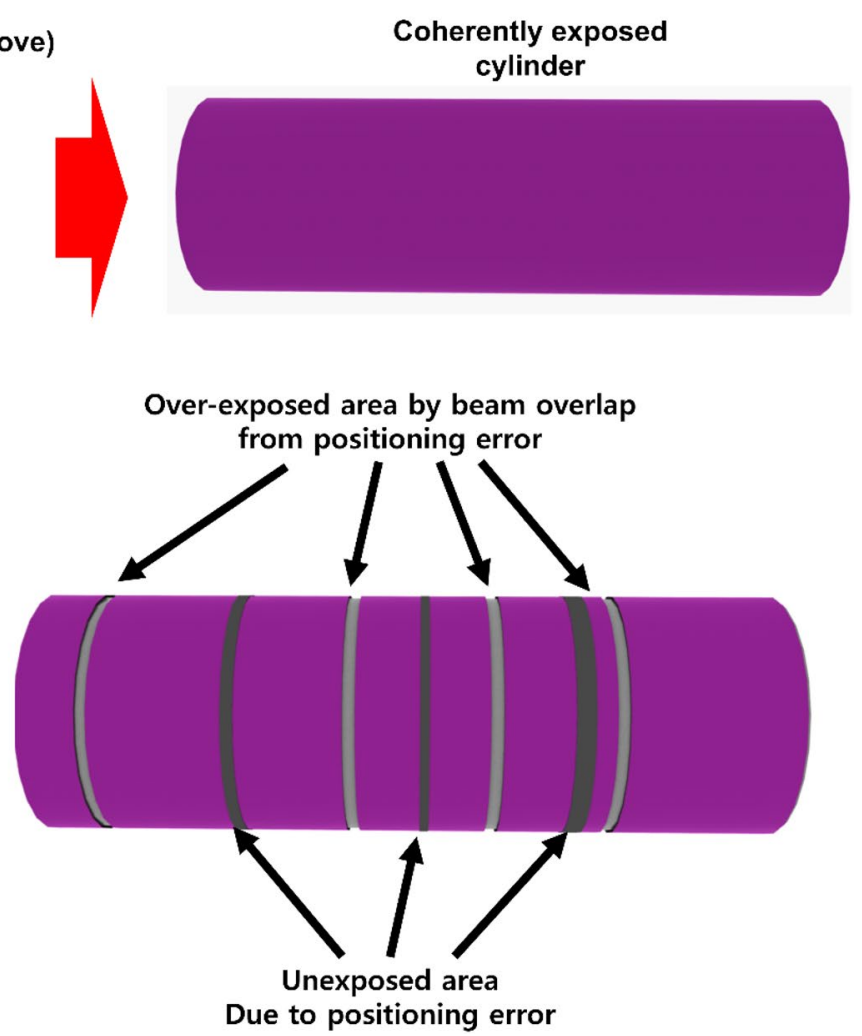

Fig. 3 Concepts of a spiral and $\mathbf{b}$ patch-to-patch processes. The black arrow in the left image indicates the beam path 


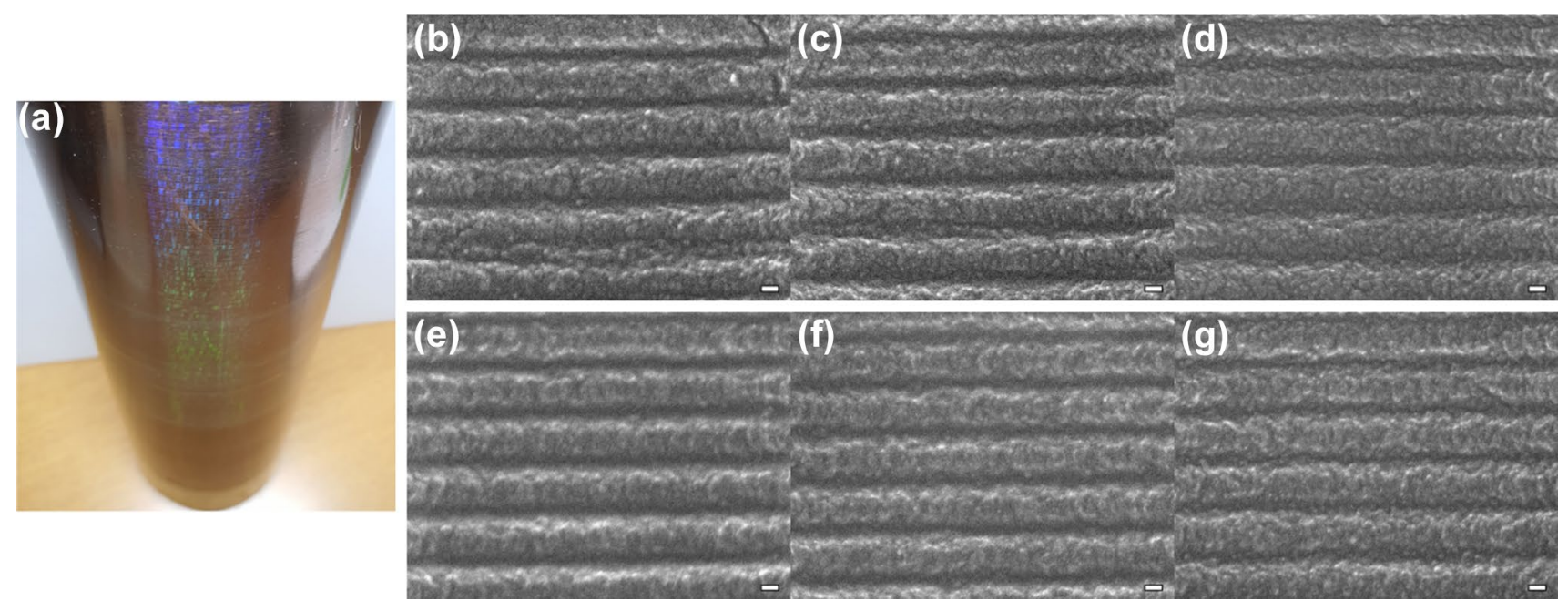

Fig. 4 Photograph and FE-SEM images of the patterns on a roll mold. a Photograph of the seamless roll mold, b-g FE-SEM images measured at six points on the PET-film-coated UV curable resin peeled off the roll mold $(\times 10,000$ magnification; scale bar, $100 \mathrm{~nm})$

depth cannot be created over the entire area. Second, the pattern cannot be replicated under constant pressure using a UV-curable resin. The third reason is related to the distance between the cylinder and optical component. We used a laser displacement sensor to measure the distance between the prism and cylinder surface; however, the displacement was variable due to the surface flatness of the cylinder, PR coating thickness, and alignment of the stage and optical system. In addition, the laser power also had errors in which very small fluctuations occurred. Despite the possibility of various errors, the shape of the pattern at six points was produced almost uniformly, and we plan a continuous manufacturing process using our seamless cylinder-shaped mold in the future.

\section{Conclusion}

In this study, we have designed an innovative laser interference exposure system and proposed a spiral process using laser interference to fabricate large-area seamless cylinder molds through continuous exposure. A PR-coated cylinder was exposed to a two-beam laser interference using a prism. Patterned-roll samples were transferred to the replicated PET films at six locations. The pattern line width was approximately $75 \mathrm{~nm}$, and the period was approximately $286 \mathrm{~nm}$. The final product, that is, the nanopatterned cylindrical mold was suitable for the continuous production of rollertype nanoimprints. It can be applied to the affordable mass production of nanotechnology products that could be used in electronics, displays, biosensors, and energy harvesting.

In the future, we aim to focus on the following aspects:
(1) Using a laser-beam homogenizer, that is, a flat-top beam, we intend to maintain both the period and depth of the pattern as constant.

(2) Pattern errors can be reduced by equipping an autofocusing system that measures and compensates for the distance between the cylinders and optical components.

(3) Using optical components that can divide the beam, such as a beam splitter and phase mask, we intend to construct a system that can change the period of the patterns in real time.

Supplementary Information The online version contains supplementary material available at https://doi.org/10.1007/s40684-022-00423-1.

Acknowledgements This work was supported by the Technology Innovation Program [N0002310] funded by the Ministry of Trade, Industry, and Energy (MOTIE, Korea) and this work was also supported by the Basic Science Research Program, through a National Research Foundation of Korea (NRF) grant funded by the Ministry of Education (2018R1D1A3B0704566214).

Author Contributions Y-WM: Primary author, conceptualization, writing-original draft, review \& editing, and investigation (overall system design/experimental design); JHP: Writing-review \& editing, and investigation (optical system design/experiments); SJL: Investigation (experimental design and experiments); JL: Investigation (experiments); SC: Investigation (experiments); BSS: Supervison.

Funding This work was supported by the Technology Innovation Program [N0002310] funded by the Ministry of Trade, Industry, and Energy (MOTIE, Korea) and this work was also supported by the Basic Science Research Program, through a National Research Foundation of Korea (NRF) Grant funded by the Ministry of Education (2018R1D1A3B07045662).

Availability of Data and Material Not applicable. 
Code Availability COMSOL, Version 5.5, Ray optics module was used for ray tracing simulation.

\section{Declarations}

Conflict of interest Not applicable.

Open Access This article is licensed under a Creative Commons Attribution 4.0 International License, which permits use, sharing, adaptation, distribution and reproduction in any medium or format, as long as you give appropriate credit to the original author(s) and the source, provide a link to the Creative Commons licence, and indicate if changes were made. The images or other third party material in this article are included in the article's Creative Commons licence, unless indicated otherwise in a credit line to the material. If material is not included in the article's Creative Commons licence and your intended use is not permitted by statutory regulation or exceeds the permitted use, you will need to obtain permission directly from the copyright holder. To view a copy of this licence, visit http://creativecommons.org/licenses/by/4.0/.

\section{References}

1. Ahn, S. H., \& Guo, L. J. (2008). High-speed roll-to-roll nanoimprint lithography on flexible plastic substrates. Advanced Materials, 20(11), 2044-2049.

2. Ahn, S. H., \& Guo, L. J. (2009). Large-area roll-to-roll and roll-toplate nanoimprint lithography: A step toward high-throughput application of continuous nanoimprinting. ACS Nano, 3(8), 2304-2310.

3. Ahn, S., Cha, J., Myung, H., Kim, S., \& Kang, S. (2006). Continuous ultraviolet roll nanoimprinting process for replicating large-scale nano-and micropatterns. Applied Physics Letters, 89(21), 213101.

4. Bagheri, S., Strohfeldt, N., Sterl, F., Berrier, A., Tittl, A., \& Giessen, H. (2016). Large-area low-cost plasmonic perfect absorber chemical sensor fabricated by laser interference lithography. ACS Sensors, 1(9), 1148-1154.

5. Cates, N., Einck, V. J., Micklow, L., Morère, J., Okoroanyanwu, U., Watkins, J. J., \& Furst, S. (2021). Roll-to-roll nanoimprint lithography using a seamless cylindrical mold nanopatterned with a highspeed mastering process. Nanotechnology, 32(15), 155301.

6. de Boor, J., Kim, D. S., \& Schmidt, V. (2010). Sub-50 nm patterning by immersion interference lithography using a Littrow prism as a Lloyd's interferometer. Optics Letters, 35(20), 3450-3452.

7. Dumond, J. J., Mahabadi, K. A., Yee, Y. S., Tan, C., Fuh, J. Y. H., Lee, H. P., \& Low, H. Y. (2012). High resolution UV roll-to-roll nanoimprinting of resin moulds and subsequent replication via thermal nanoimprint lithography. Nanotechnology, 23(48), 485310.

8. Ishizawa, N., Idei, K., Kimura, T., Noda, D., \& Hattori, T. (2008). Resin micromachining by roller hot embossing. Microsystem Technologies, 14(9), 1381-1388.

9. Kim, M., Park, C., Je, S., Jang, H., Joo, C., \& Kang, S. (2018). Realtime compensation of simultaneous errors induced by optical phase difference and substrate motion in scanning beam laser interference lithography system. IEEE/ASME Transactions on Mechatronics, 23(4), 1491-1500.

10. Kim, P. K., Chung, S., Ha, T., \& Jeong, M. Y. (2020). The fabrication of a cylindrical nano mold based on UV photolithography. Science of Advanced Materials, 12(3), 407-411.

11. Komatsu, K., Abe, M., Ito, N., \& Matsui, S. (2019). R2R nanopatterning technology using $250 \mathrm{~mm}$-wide seamless roller mold. In: Paper presented at the 2019 International Conference on Electronics Packaging (ICEP), pp 163-166.

12. Lee, Y., Chen, P., \& Lin, H. (2009). Fabrication of seamless roller mold with excimer laser direct writing technology. In: Paper
Presented at the 2009 4th IEEE International Conference on Nano/ Micro Engineered and Molecular Systems, pp. 767-770.

13. Lim, H., Jung, S., Ahn, J., Choi, K., Kim, G., Kwon, S., \& Lee, J. (2020). System for fabrication of large-area roll molds by step-andrepeat liquid transfer imprint lithography. Materials, 13(8), 1938.

14. Ma, Y., Park, J. H., Yun, D. H., Gwak, C., \& Shin, B. S. (2019). Double exposure laser interference lithography for pattern diversity using ultraviolet continuous-wave laser. Journal of the Microelectronics and Packaging Society, 26(2), 9-14.

15. Nguyen, H., \& Lee, Y. (2019). Step and scanning lithography for patterning seamless microstructures on the inner surface of a hollow roller. Journal of Micromechanics and Microengineering, 29(10), 105012.

16. Ojima, K., Saito, M., Unno, N., \& Taniguchi, J. (2016). Fabrication characteristics of a line-and-space pattern and a dot pattern on a roll mold by using electron-beam lithography. Journal of Advanced Mechanical Design, Systems, and Manufacturing, 10(5), JAMDSM0074.

17. Park, J. H., Yun, D. H., Ma, Y. W., Gwak, C. Y., \& Shin, B. S. (2020). Prism-based laser interference lithography system for simple multibeam interference lithography. Science of Advanced Materials, 12(3), 398-402.

18. Rank, A., Lang, V., \& Lasagni, A. F. (2017). High-speed roll-toroll hot embossing of micrometer and sub micrometer structures using seamless direct laser interference patterning treated sleeves. Advanced Engineering Materials, 19(11), 1700201.

19. Sidharthan, R., \& Murukeshan, V. M. (2011). Pattern definition employing prism-based deep ultraviolet lithography. Micro \& Nano Letters, 6(3), 109-112.

20. So, J., \& Bae, W. (2018). Fabrication of superhydrophobic metallic surface by wire electrical discharge machining for seamless roll-toroll printing. Metals, 8(4), 228.

21. Striegel, A., Schneider, M., Schneider, N., Benkel, C., \& Worgull, M. (2018). Seamless tool fabrication for Roll-to-Roll microreplication. Microelectronic Engineering, 194, 8-14.

22. Takano, Y., \& Taniguchi, J. (2018). Fabrication of the grid pattern on a roll mold by electron beam direct writing. Microelectronic Engineering, 193, 41-46.

23. Tan, L. K., \& Ho, A. Y. Y. (2016). Sub-100 nm features seamless roller mold using anodization and atomic layer deposition, for rollto-roll nanoimprint lithography. Journal of Vacuum Science \& Technology B, Nanotechnology and Microelectronics: Materials, Processing, Measurement, and Phenomena, 34(5), 051601.

24. Tseng, S. C., Peng, W. Y., Hsieh, Y. F., Lee, P. J., \& Lai, W. L. (2010). Electron beam lithography on cylindrical roller. Microelectronic Engineering, 87(5-8), 943-946.

25. Unno, N., Taniguchi, J., \& Ishikawa, K. (2011). Fabrication of a seamless roll mold using inorganic electron beam resist with postexposure bake. Journal of Vacuum Science \& Technology B, Nanotechnology and Microelectronics: Materials, Processing, Measurement, and Phenomena, 29(6), 06FC06.

26. Vala, M., \& Homola, J. (2016). Multiple beam interference lithography: A tool for rapid fabrication of plasmonic arrays of arbitrary shaped nanomotifs. Optics Express, 24(14), 15656-15665.

27. Wolf, A. J., Hauser, H., Kübler, V., Walk, C., Höhn, O., \& Bläsi, B. (2012). Origination of nano-and microstructures on large areas by interference lithography. Microelectronic Engineering, 98, 293-296.

28. Xie, Q., Hong, M. H., Tan, H. L., Chen, G. X., Shi, L. P., \& Chong, T. C. (2008). Fabrication of nanostructures with laser interference lithography. Journal of Alloys and Compounds, 449(1-2), 261-264.

29. Xu, J., Wang, Z., Zhang, Z., Wang, D., \& Weng, Z. (2014). Fabrication of moth-eye structures on silicon by direct six-beam laser interference lithography. Journal of Applied Physics, 115(20), 203101.

30. Zhang, C., Chen, R., Yang, L., Wu, H., Ji, S., Zhang, J., Zhou, L., Ye, H., Wu, S., \& Zhang, J. (2020). Structural color surface on transparent PDMS fabricated by carbon-assisted laser interference 
lithography for real-time quantification of soft actuators motion. ACS Applied Materials \& Interfaces, 12(40), 45641-45647.

31. Park, J. H., Yun, D. H., Ma, Y. W., Gwak, C. Y., Je, G., \& Shin, B. S. (2018). Laser interference lithography on non-planar surface for roll-to-roll process. In: Paper presented at the Laser-Based Microand Nanoprocessing XII, $10520105201 \mathrm{D}$.

Publisher's Note Springer Nature remains neutral with regard to jurisdictional claims in published maps and institutional affiliations.

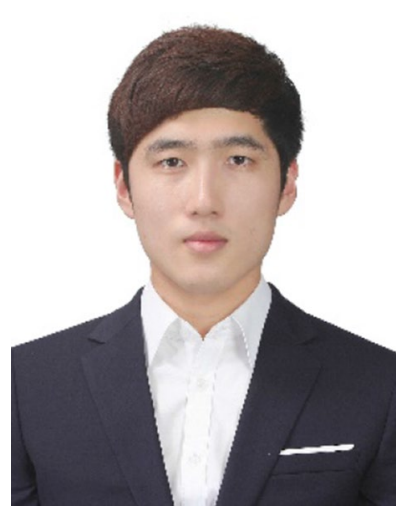

Yong-Won Ma received M.E. in mechatronics from the Pusan National University. He is currently a $\mathrm{PhD}$ candidate at the Pusan National University, focusing on laser-based micro/ nanofabrication systems towards optic applications and sensors. His current research interests include the periodic nanopatterning, additive manufacturing and fabrication of carbon materials decorated nanoparticles.
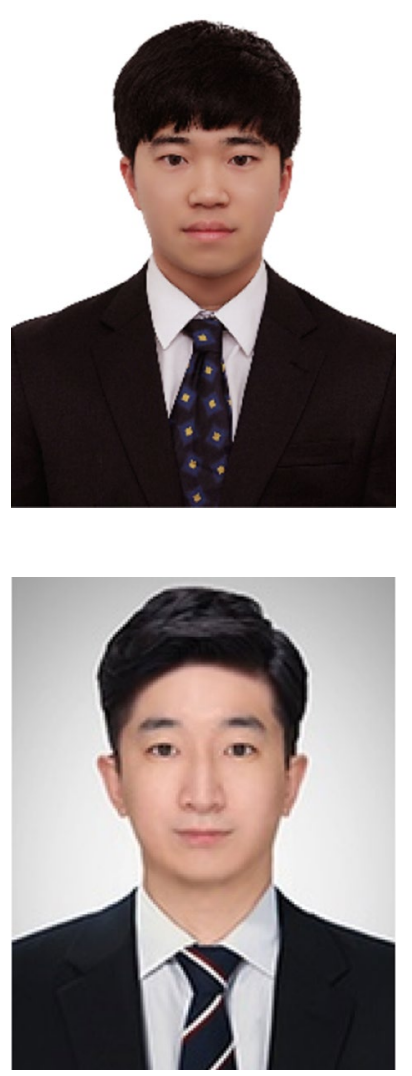

Jun Han Park received his B.Sc. in optics and mechatronics engineering from the Pusan National University. He is currently a PhD candidate at the Pusan National University, focusing on laser application for micro/nanofabrication. His main research demonstrates cost-effective fabrication of sub-micrometer structures using laser interference.

Sung Jae Lee received B.Sc. in Department of Optics and mechatronics Engineering, College of Nanoscience and Nanotechnology, Pusan National University (PNU). He received a diploma Cogno-Mechatronics Engineering (PNU) and Master degree at Department of Applied Science for Electronics and Materials in Kyushu university. He worked on LIMIT lab and have researched laser Interference lithography focusing on fabrication of uniform interference pattern, fabrication of nano

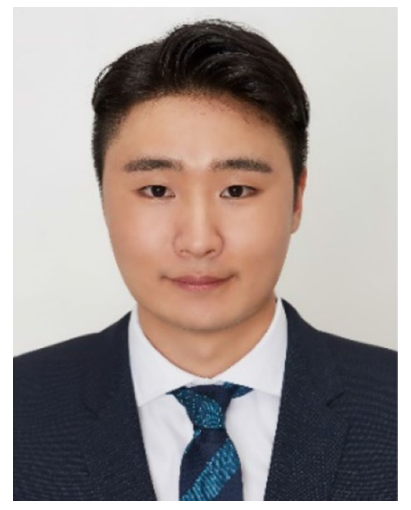

Jeonghoon Lee is currently Master degree student at CognoMechatronics Engineering of Pusan National University (PNU) of Korea. Also, he proceeds interdisciplinary Master degree course from Kyushu University of Japan. He received bachelor diploma from Optics and Mechatronics Engineering of PNU. His research area is focused on the laser fabrication and its application using UV wavelength laser. He participate projects which conduct laser interference lithography for nano patterning on roller mold and laser induced graphene(LIG) and metal oxide nano particle synthesis using $355 \mathrm{~nm}$ laser.

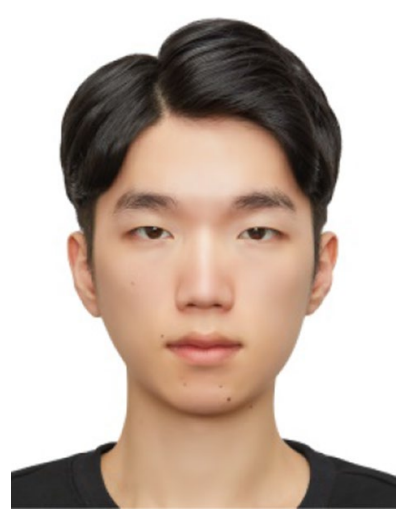

Suchan Cho received the B.Sc. with distinction in Optics and Mechatronics Engineering from the Pusan National University, Busan, Republic of Korea, in 2021. He is currently studying for a master's degree in CognoMechatronics Engineering at the Pusan National University. His work primarily involves sensor fabrication and laser processing. Whilst studying for his master's degree, he has been sponsored by a Korea Institute for the Advancement of Technology (KIAT) grant.

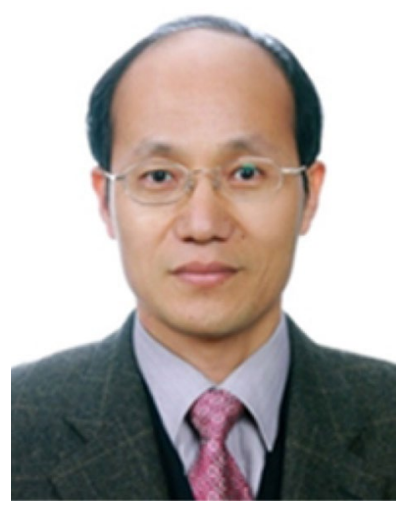

Bo Sung Shin received the Ph.D. in mechanical engineering from the Korea Advanced Institute of Science and Technology (KAIST). After being with Department of Ordnance Engineering in Korea Military Academy as a Full-time professor for 3 years, he joined Korea institute of machinery \& materials (KIMM) as a director in the Nano process group. Since 2012, he has been a lecturer in the Department of Optics and Mechatronics Engineering at the Pusan National University, Pusan, Republic of Korea. His work primarily involves finite element modelling of magnetic and mechanical systems, and laser processing system design.

pattern on cylinder mold. 